\title{
Water Capture Mechanisms at Zeolitic Imidazolate Framework Interfaces
}

Jackson C. Wagner ${ }^{1}$, Kelly M. Hunter ${ }^{1}$, Francesco Paesani ${ }^{1,2 *}$, Wei Xiong ${ }^{1,2,3 *}$

${ }^{1}$ Department of Chemistry and Biochemistry, University of California, San Diego, CA, USA, 92093

${ }^{2}$ Materials Science and Engineering Program, University of California, San Diego, CA, USA, 92093

${ }^{3}$ Department of Electrical and Computer Engineering, University of California, San Diego, CA, USA, 92093

\section{Supplementary Information}

S1. Method and Materials

S2. VSFG control and characterization experiments

S3. DRIFTS spectral fitting methods and results

S4. VSFG OD extraction methods

S5. VSFG data analysis results

S6. Simulation methods and additional results 


\section{S1. Method and Materials}

Spatially-resolved VSFG spectrometer.

To reduce the contribution from scattering surfaces and confine our analysis to only single crystals a reflective microscope geometry was used, a schematic of the instrument can be found in SI Fig 1. The signal and idler of a Ti:Sapphire (Coherent, Astrella) pumped OPA (Light Conversion, TOPAS Prime) were temporally and spatially combined at a DFG crystal to generate a Mid-IR (MIR) pulse. The generated MIR was directed through a delay stage to the back of a customized dichroic mirror that is transmissive to MIR and reflective to Near-IR. The residual $800 \mathrm{~nm}$ from the OPA was directed through a folded $4 \mathrm{f}$ pulse shaper to spectrally condition the beam as an upconversion. The upconversion was then spatially overlapped with the MIR beam using the customized dichroic mirror and both beams were directed to a purely reflective infinity corrected Schwarzschild objective to be focused on the sample area. The reflected VSFG signal is then collected with the same reflective objective and focused into the entrance slit of a Monochromator which dispersed the signal into spectra and projected it to a CCD to be analyzed (Andor, Shammrock 500i, Newton CCD).

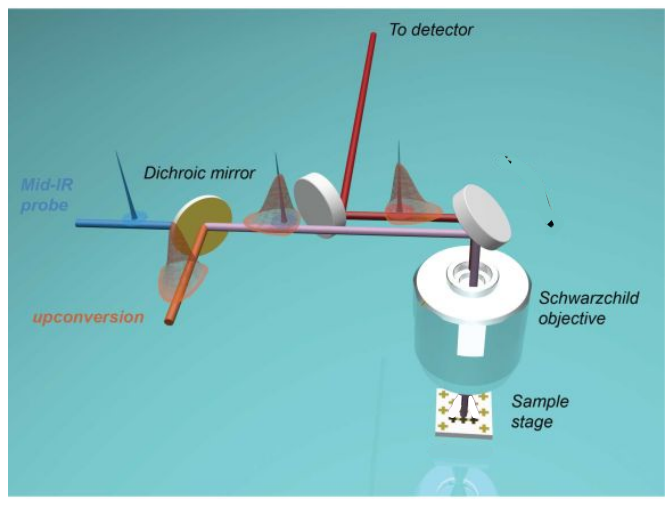

SI Figure 1 Core schematic of VSFG reflection microscope used to study ZIF-90 at a micron scale.

To create an environment with a controlled humidity of $\mathrm{D}_{2} \mathrm{O}$ for the spatially resolved VSFG microscope, an environmental chamber (SI Figure 2) was fabricated from aluminum with a $\mathrm{CaF}_{2}$ window which is transparent to the upconversion and MIR used. The beam trajectory of the incoming NIR and MIR beams and outgoing VSFG signal beams are shown as well as the flow path of humidified air over the sample area.

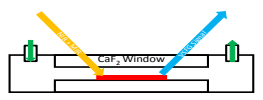

SI Figure 2 VSFG environmental chamber schematic cross section with the air flow labeled in green, NIR and MIR input beam paths in orange, VSFG signal beam path in blue and sample area in red. 


\section{Preparation and Characterization of ZIF-90}

ZIF-90 nanocrystals were synthesized using techniques detailed in literature. In brief, ZIF-90 was synthesized by fully dissolving imidazole-2-carboxaldehyde (ICA) in N,N-dimethyl-formamide (DMF) with heat as described in ref $1 .{ }^{1}$ The ICA/DMF solution was cooled to room temperature, and pyridine was added. A solution of zinc nitrate hexahydrate in methanol was poured into the ICA/ pyridine/DMF solution rapidly and mixed overnight. ZIF-90 crystals were collected through four cycles of centrifugation with neat DMF. The collected product was then washed with DMF and dried.

ZIF-90 microcrystals were synthesized using techniques detailed in literature. In brief, ZIF-90 was synthesized by fully dissolving ICA and zinc nitrate in N,N-dimethyl-formamide (DMF) with heat as described in ref $2 .{ }^{2}$ Once fully dissolved, the solution was removed from heat and allowed to sit undisturbed for 24 hours at room temperature. The crystals were collected with a metal spatula, rinsed centrifugally with DMF twice and rinsed with methanol thrice and stored in methanol until use.

Powder X-Ray Diffraction (PXRD) and Scanning Electron Microscopy (SEM) characterization techniques were applied to the synthesized ZIF-90 powders to ensure the products purity. When the experimentally obtained PXRD pattern for ZIF-90 is compared with that of the calculated pattern (SI Figure 3), both patterns are nearly identical. The SEM images which can be found in the main body text further confirm that ZIF-90 was properly synthesized because of the reported SOD topology.

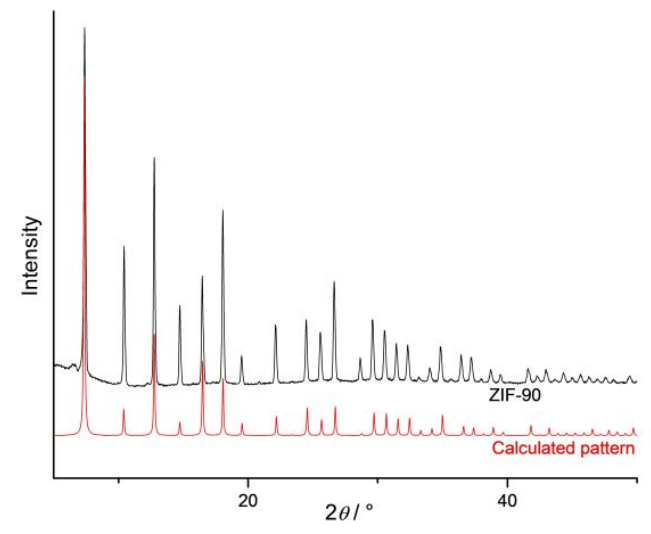

SI Figure 3 Theoretical PXRD of ZIF-90 (red) and experimental PXRD of ZIF-90 (black) confirming the synthesis.

\section{DRIFTS of ZIF-90}

Diffuse reflectance Infrared Fourier Transform Spectroscopy (DRIFTS), a vibrational spectroscopic technique for powdered samples was performed on ZIF-90 crystals. The DRIFTS accessory (DiffusIR, Pike Technologies Inc) was mounted to a Nicolet IS10 FTIR. The ZIF-90 crystals were ground with dry $\mathrm{KBr}$ powder to form a well-mixed and uniform sample at 5\% ZIF90 by mass. A pure KBr powder was made at the same time as well. The mixed KBr/ZIF-90 powder was placed in the sample holder of the accessory and the pure $\mathrm{KBr}$ standard was placed 
inside as well. The DRIFTS accessory was then purged using a system outlined in SI Figure 4. Here, dry air is bubbled through pure $\mathrm{D}_{2} \mathrm{O}$ to generate nearly saturated humidified air which can then be mixed with dry air to produce a range of humidity. This same system was used to supply humidified air to the VSFG environmental chamber in SI Figure 2.

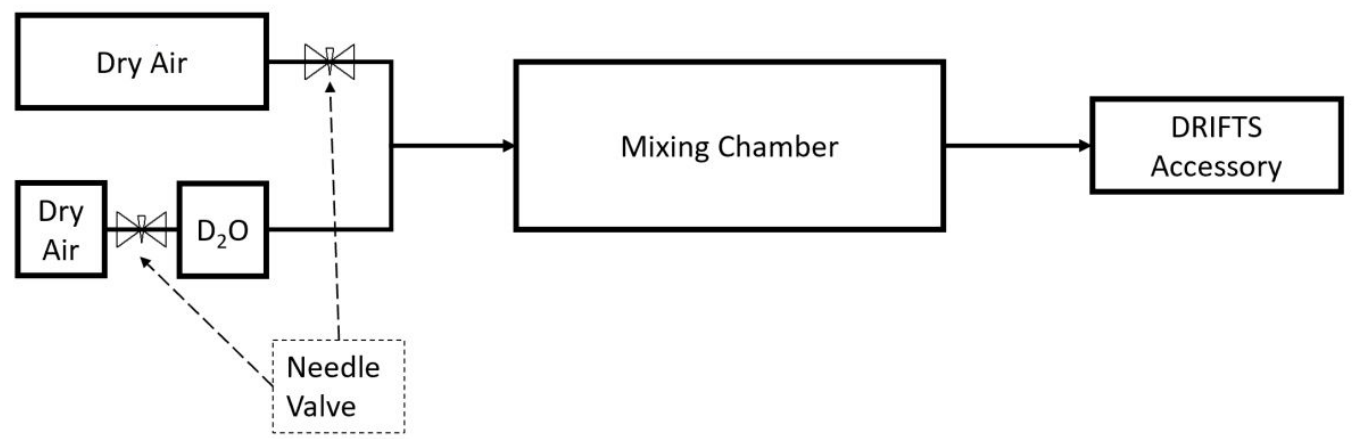

SI Figure 4 Schematic of purging system used to deliver $\mathrm{H}_{2} \mathrm{O}$ and $\mathrm{D}_{2} \mathrm{O}$ humidifies environments to DiffusIR DRIFTS accessory and VSFG environmental cell.

After the humidity stabilized at a RH setpoint of interest, spectra of the sample and $\mathrm{KBr}$ standard were taken.

$$
F\left(R_{\infty}\right)=\frac{\left(1-R_{\infty}\right)^{2}}{2 R_{\infty}}=\frac{k}{s}
$$

The in-software correction was applied (Eq. 1) in which $\mathrm{R}_{\infty}$ is the reflectance at infinity, $\mathrm{k}$ is the collected signal for the sample in the DRIFTS accessory and $\mathrm{s}$ is the signal of the $\mathrm{KBr}$ standard which, is a perfect absorber and scatterer. This data analysis technique removes the large contribution of scattering to the complexity of further analysis. The results from the collection and scattering removal are shown fit in SI Figure 10. 


\section{S2. VSFG control experiments}

To rule out erroneous interpretations of the results a few control experiments were performed. SI Figure 5 shows data of a nonlinear crystal GaAs inside the environmental control chamber. Even with a high humidity applied to the environmental cell the nonlinear signal from GaAs does not change ruling the potential for condensed water to have on the analysis.

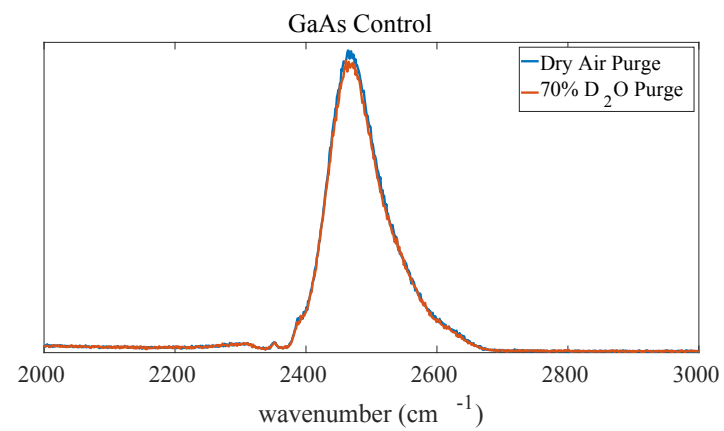

SI Figure 5 High $\mathrm{D}_{2} 0$ humidity purge spectra of GaAs compared to that dry air. This proves the atmospheric purge does not affect the VSFG spectral lineshape due to moisture condensing on interfaces of the environmental cell.

Additional control experiments using $\mathrm{H}_{2} \mathrm{O}$ rather than $\mathrm{D}_{2} \mathrm{O}$ were performed to ensure the dip feature observed with $\mathrm{D}_{2} \mathrm{O}$ was indeed unique. Results from this control experiment (SI Figure 6) show that although the signal does decrease under high humidity, which we attribute to the change in the index of refraction upon hydration, the spectral line shape is consistent and no dip feature appears.

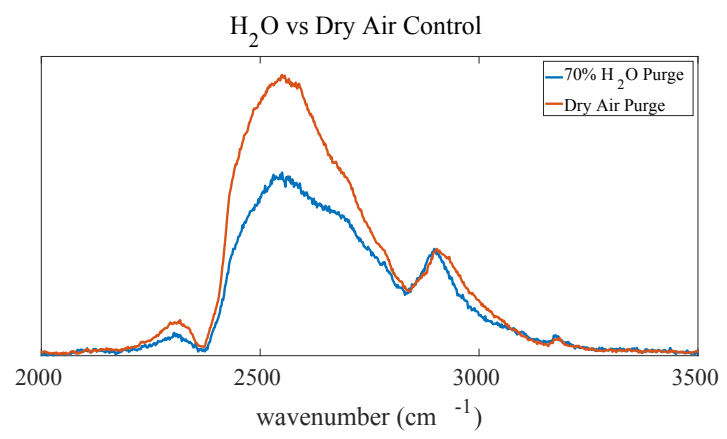

SI Figure 6 VSFG of ZIF-90 under dry air and $\mathrm{H}_{2} \mathrm{O}$ humidified environmental conditions. Attenuation of the $\mathrm{H}_{2} \mathrm{O}$ environmental signal is attributed to the change in the index of refraction of the ZIF-90 crystal upon hydration. 


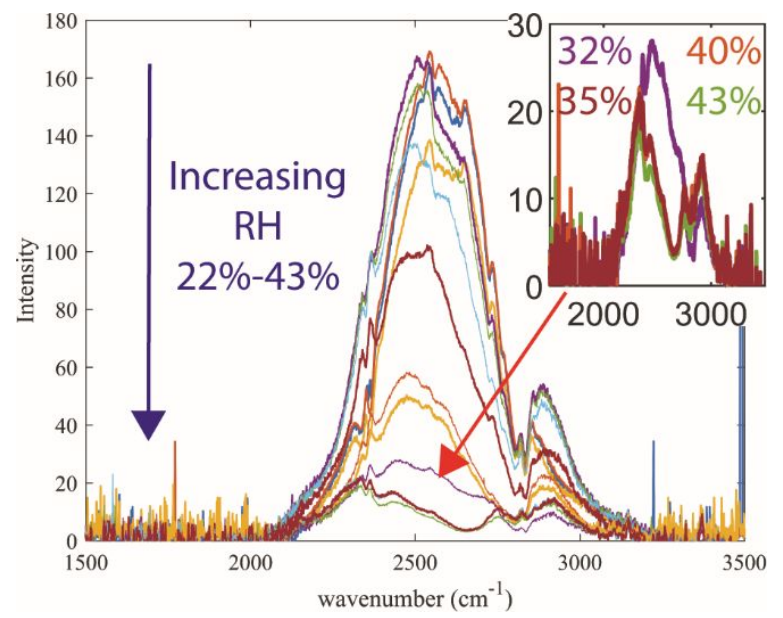

SI Figure 7 Choice of RH purging region. Using the approximate point of inflection from the adsorption isotherm of ZIF-90, it is apparent that there is virtually no signal or lineshape change following this point of inflection around 30\%-35\%. The results from this characterization experiment guided our selection of the RH dependent data of main text Fig. 3 and 4.

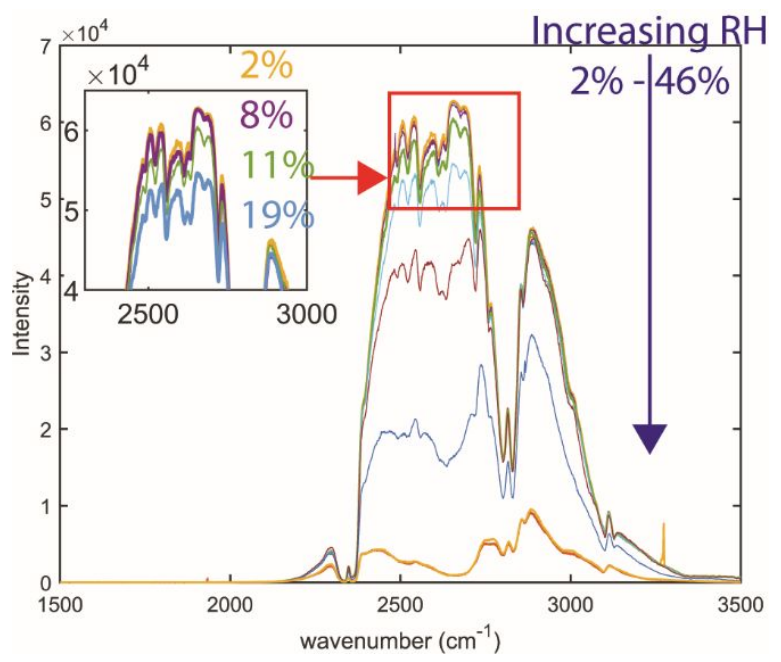

SI Figure 8 Full humidity range data showing that even at lower humidity, a similar drop in intensity is observed as to that of higher intensity. This drop in intensity before the formation of the molecular feature is due to the introduction of polarizable $\mathrm{D}_{2} \mathrm{O}$ into ZIF-90 which increases the overall index of refraction leading to either a defocus or change in the Fresnel factor, reducing the overall intensity. The overall nonresonant signal lineshape differs from SI Figure 7 because of a different MOF sample, which changes the scattering profile of the signal, and a different IR and upconversion time-delay used here. However, this difference in nonresonant background does not change the conclusion drawn from this plot - at low RH region, only the overall SFG intensity changes but no resonant feature developed when increasing RH (till $30 \%)$.

\section{Reduction in Overall VSFG Signal}

Using the Fresnel equations (Eq. 2,3,4) and keeping the incident, transmitted beam angles constant, $\Omega_{\mathrm{i}}$ and $\Omega_{\mathrm{t}}$ respectively, and first index of refraction, $\mathrm{n}_{1}$, we see that after an increase in the index of refraction of the MOF upon hydration $\left(\mathrm{n}_{2}\right)$ all calculated Fresnel factor $\left(\mathrm{L}_{\mathrm{ii}}\right)$ decreases in value. The $\mathrm{L}_{\mathrm{xx}}, \mathrm{L}_{\mathrm{yy}}$, and $\mathrm{L}_{\mathrm{zz}}$ Fresnel projections are grouped as a single frequency 
dependent Fresnel factor, $\mathrm{L}(\omega)$, which is related to second order nonlinear susceptibility through Eq. 5 where $\hat{e}\left(\omega_{i}\right)$ are the unit vector electric field for the signal, NIR and MIR beams, $\chi_{e f f}^{(2)}$ is the effective second order nonlinear susceptibility and $\chi^{(2)}$ is the second order nonlinear susceptibility.

$$
\begin{aligned}
L_{x x} & =\frac{2 n_{1} \cos \Omega_{t}}{n_{2} \cos \Omega_{i}+n_{1} \cos \Omega_{t}} \\
L_{y y} & =\frac{2 n_{1} \cos \Omega_{t}}{n_{1} \cos \Omega_{i}+n_{2} \cos \Omega_{t}} \\
L_{z z} & =\frac{2 n_{2} \cos \Omega_{t}}{n_{2} \cos \Omega_{i}+n_{1} \cos \Omega_{t}} \\
\chi_{e f f}^{(2)}=[\hat{e}(\omega) * L(\omega)] * & \chi^{(2)}:\left[L\left(\omega_{1}\right) * \hat{e}\left(\omega_{1}\right)\right]\left[L\left(\omega_{2}\right) * \hat{e}\left(\omega_{2}\right)\right]
\end{aligned}
$$

\section{S3. DRIFTS spectral fitting methods and results}

The data obtained from DRIFTS and analyzed using Eq. 1 are summarized in SI Figure 9. In all cases, spectra were fit using 3 gaussians (Eq. 2). As the supplied humidity increases, the intensity of the Fermi resonance (red peak), asymmetric (yellow peak) and symmetric (purple peak) increase monotonically.

$$
S_{\text {total }}=A_{1} e^{\left(-\frac{\left(w-B_{1}\right)}{C_{1}}\right)^{2}}+A_{2} e^{\left(-\frac{\left(w-B_{2}\right)}{C_{2}}\right)^{2}}+A_{3} e^{\left(-\frac{\left(w-B_{3}\right)}{C_{3}}\right)^{2}}
$$



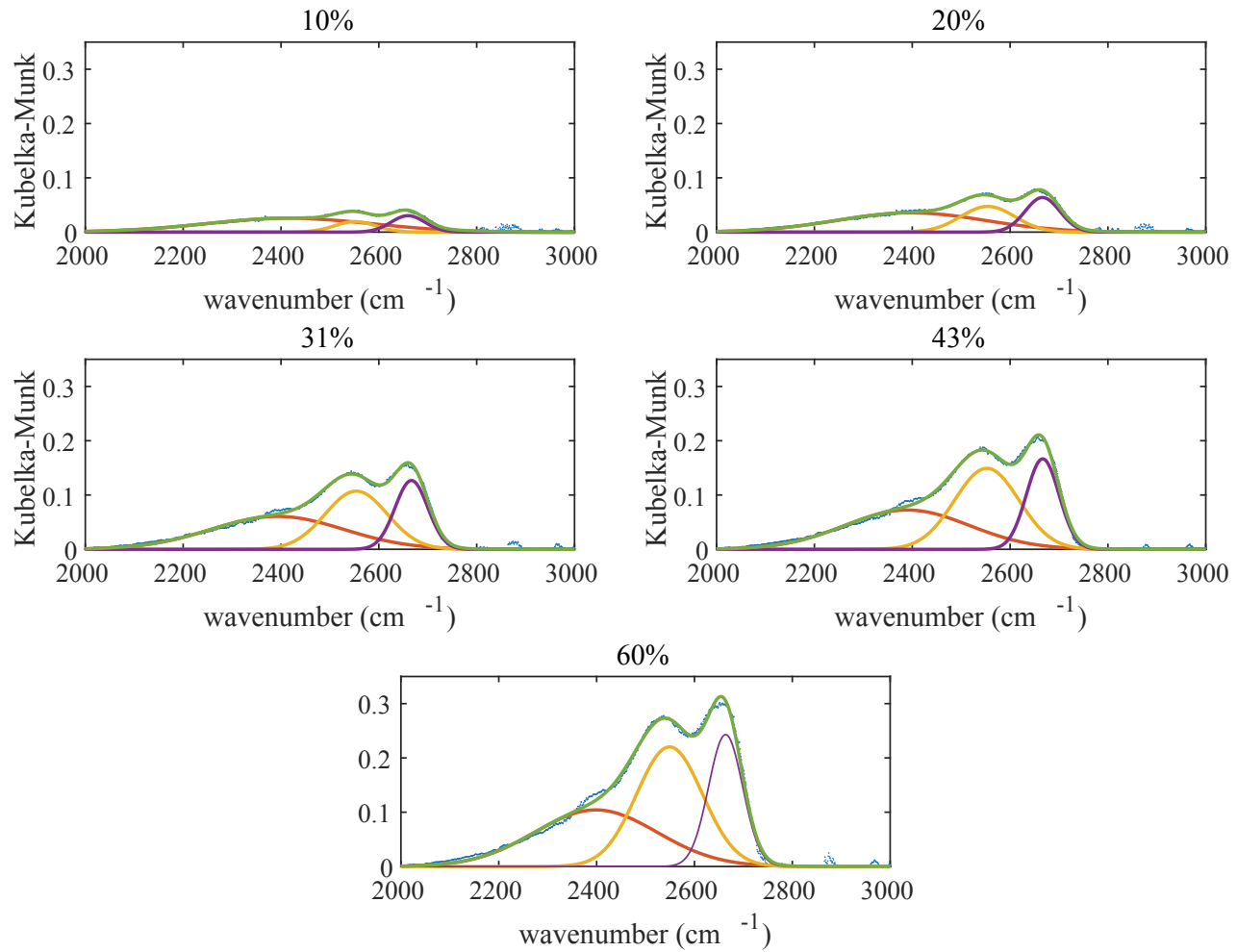

SI Figure 9 DRIFTS spectra from RH purges ranging from 10\%-60\%. Compared with VSFG spectra in SI Fig 6, spectra show 3 clear features corresponding to the fermi resonance (red) and symmetric (yellow) and asymmetric stretches (purple) of water in the MOF pore.

SI Table 1 95\% confidence interval for fitted DRIFTS spectra in SI Figure 7.

\begin{tabular}{|c|c|c|c|c|c|c|}
\hline & \multicolumn{3}{|c|}{$10 \% \mathrm{RH}$} & \multicolumn{3}{c|}{ 20\%RH } \\
\cline { 2 - 7 } & Lower & Equilibrium & Upper & Lower & Equilibrium & Upper \\
\hline a1 & 0.02918 & 0.03019 & 0.0312 & 0.06152 & 0.06368 & 0.06583 \\
\hline b1 & 2658 & 2660 & 2662 & 2665 & 2667 & 2668 \\
\hline c1 & 46.96 & 49.37 & 51.78 & 48.42 & 50.14 & 51.86 \\
\hline a2 & 0.01824 & 0.01923 & 0.02022 & 0.04484 & 0.04746 & 0.05007 \\
\hline b2 & 2549 & 2552 & 2555 & 2552 & 2555 & 2558 \\
\hline c2 & 54.35 & 58.97 & 63.59 & 73.53 & 78.43 & 83.33 \\
\hline a3 & 0.0247 & 0.02516 & 0.02562 & 0.03462 & 0.03555 & 0.03649 \\
\hline b3 & 2413 & 2418 & 2424 & 2386 & 2396 & 2407 \\
\hline c3 & 237.2 & 242.6 & 248.1 & 203.9 & 213 & 222 \\
\hline & \multicolumn{7}{|c|}{$31 \% R H$} & & $43 \%$ RH & \\
\cline { 2 - 7 } & Lower & Equilibrium & Upper & Lower & Equilibrium & Upper \\
\hline a1 & 0.1223 & 0.1267 & 0.1311 & 0.158 & 0.2666 & 0.1751 \\
\hline b1 & 2666 & 2667 & 2668 & 2665 & 2667 & 2668 \\
\hline c1 & 46.08 & 47.46 & 48.84 & 45.32 & 47.19 & 49.05 \\
\hline a2 & 0.09977 & 0.107 & 0.1142 & 0.1333 & 0.1489 & 0.1645 \\
\hline b2 & 2552 & 2554 & 2557 & 2550 & 2553 & 2556 \\
\hline
\end{tabular}




\begin{tabular}{|c|c|c|c|c|c|c|}
\hline c2 & 84.46 & 89.83 & 95.21 & 85.18 & 93.33 & 101.5 \\
\hline a3 & 0.05793 & 0.06052 & 0.06312 & 0.06659 & 0.07241 & 0.07822 \\
\hline b3 & 2378 & 2993 & 2408 & 2365 & 2391 & 2416 \\
\hline \multirow[t]{3}{*}{ c3 } & 180.2 & 192.2 & 204.1 & 160.4 & 180.4 & 200.3 \\
\hline & \multicolumn{3}{|c|}{$60 \% \mathrm{RH}$} & & & \\
\hline & Lower & Equilibrium & Upper & & & \\
\hline a1 & 0.2321 & 0.2429 & 0.2536 & & & \\
\hline b1 & 0.2203 & 2664 & 2665 & & & \\
\hline c1 & 2550 & 49.28 & 50.84 & & & \\
\hline$a 2$ & 0.1969 & 0.2203 & 0.2437 & & & \\
\hline b2 & 2547 & 2550 & 2553 & & & \\
\hline$c 2$ & 88.08 & 95.3 & 102.5 & & & \\
\hline a3 & 0.09423 & 0.104 & 0.1137 & & & \\
\hline b3 & 2374 & 2399 & 2425 & & & \\
\hline c3 & 158.5 & 176.5 & 194.4 & & & \\
\hline
\end{tabular}

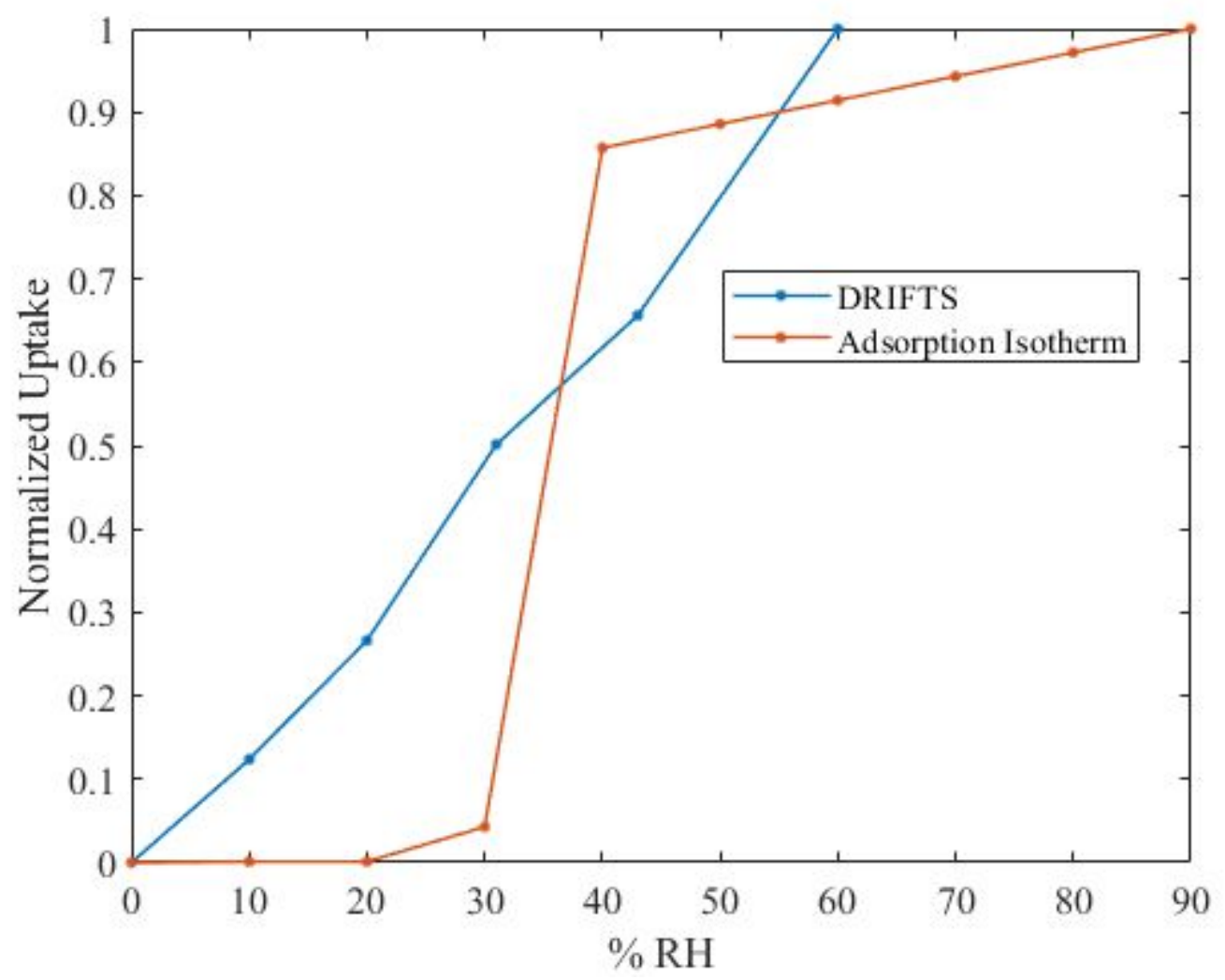

SI Figure 10 Peak integration of the DRIFTS peak centered around $2660 \mathrm{~cm}^{-1}$ vs RH. The plot contrasts that of adsorption isotherms. DRIFTS is more sensitive to lower concentrations for highly IR absorbing modes, as at high concentration, the IR absorber will effectively decrease the pathlength in DRIFTS, which explains why there is appreciable sensitivity at lower humidity, but cannot proportionally reflect the onset of water update. 


\section{S4. VSFG OD extraction methods}

To extract the molecular response that appears as a dip feature in hydrated spectra we start with the symbolic equation representing the nonresonant response from the dry air purge ZIF-90 (Eq. 7). Where $S_{N R}$ is the nonresonant second order optical response of ZIF- $90, \chi_{N R}{ }^{(2)}$ is the nonresonant second order nonlinear optical susceptibility of ZIF-90 and $\mathrm{f}(\mathrm{RH})$ is the arbitrary function describing the change in absolute signal intensity due to changes in the index of refraction as a function of $\mathrm{RH}$. When $\mathrm{RH}=0, \mathrm{f}(\mathrm{RH})=1$.

$$
S_{N R}(R H)=\left|f(R H) \chi_{N R}^{(2)}\right|^{2} I_{1} I_{2}
$$

Taking the square root of $\mathrm{S}_{\mathrm{NR}}$ (highlighted in step 1 of $\mathbf{S I}$ Figure 10) at $\mathrm{RH}=0$ we get,

$$
\sqrt{S_{N R}(0)}=\left|\chi_{N R}^{(2)}\right| \sqrt{I_{1} I_{2}}
$$

The symbolic equation for the signal of ZIF-90 under hydrated conditions can determined similarly and is shown in Eq. 9 where, $\chi_{R}{ }^{(2)}$ is the resonant second order nonlinear susceptibility.

$$
S_{S F G}(R H)=\left[\left|f(R H) \chi_{R}^{(2)}\right|^{2}+\left|f(R H) \chi_{N R}^{(2)}\right|^{2}+2 R e\left(f(R H)^{2} \chi_{R}^{(2)} \chi_{N R}^{*}(2)\right)\right] I_{1} I_{2}
$$

We note that Eq. 9 can only be used for spectra that include both nonresonant and resonant OD features. $\chi_{R}{ }^{(2)}$ is assumed to be negligible and will be neglected. Providing the following substitutions,

$$
\begin{gathered}
\chi_{R}^{(2)}=A_{R}(w) e^{i \varphi_{R}(w)} \\
\chi_{N R}^{(2)}=A_{N R} e^{i \varphi_{N R}} \\
\left|\chi_{N R}^{(2)}\right|=A_{N R} \\
\chi_{R}^{(2)} \chi_{N R}^{(2)}=A_{R}(w) A_{N R} e^{i \Delta \varphi(w)} \\
\operatorname{Re}\left(\chi_{R}^{(2)} \chi_{N R}^{(2)}\right)=A_{R}(w) A_{N R} \cos (\Delta \varphi(w))
\end{gathered}
$$

Where $A_{R}(w)$ and $A_{N R}$ are functions describing the VSFG OD response and nonresonant responses of ZIF-90 respectively, $\varphi_{R}(w)$ and $\varphi_{N R}$ are the phases of the resonant OD response and nonresonant signal response of ZIF-90 respectively and $\Delta \varphi(w)$ is the phase difference between $\varphi_{\mathrm{R}}(\mathrm{w})$ and $\varphi_{\mathrm{NR}}$.

Results in the updated Eq. 8 and Eq. 9 ,

$$
\begin{gathered}
\sqrt{S_{N R}(0)}=A_{N R} \sqrt{I_{1} I_{2}} \\
S_{S F G}(R H)=\left[f(R H)^{2} A_{N R}^{2}+2 f(R H)^{2} A_{R}(w) A_{N R} \cos (\Delta \varphi(w))\right] I_{1} I_{2}
\end{gathered}
$$

Then we divide Eq. 9b by Eq. 8b (results shown in SI Figure 10) to get, 


$$
\begin{aligned}
\frac{S_{S F G}(R H)}{\sqrt{S_{N R}(0)}} & \\
& =\frac{\left[f(R H)^{2} A_{N R}^{2}+2 f(R H)^{2} A_{R}(w) A_{N R} \cos (\Delta \varphi(w))\right] I_{1} I_{2}}{A_{N R} \sqrt{I_{1} I_{2}}}= \\
& {\left[A_{N R}+2 A_{R}(w) \cos (\Delta \varphi(w))\right] f(R H)^{2} \sqrt{I_{1} I_{2}} }
\end{aligned}
$$

Since $30.4 \%$ was the highest humidity before the appearance of the OD feature we divide Eq. 7 , with $\mathrm{f}(\mathrm{RH} \neq 0)$ by $\mathrm{f}(\mathrm{RH}=0)$ to get,

$$
\frac{S_{S F G}(30.4 \%)}{\sqrt{S_{N R}}}=\frac{f(30.4 \%)^{2} A_{N R}^{2} I_{1} I_{2}}{A_{N R} \sqrt{I_{1} I_{2}}}=f(30.4 \%)^{2} A_{N R} \sqrt{I_{1} I_{2}}
$$

All spectra were normalized to their $\mathrm{CH}$ resonant modes at $\sim 2820 \mathrm{~cm}^{-1}$ to account for the effects of the $f(R H)$ change below $30.4 \%$, which makes $f(R H)$ a constant after the normalization. Subtracting Eq. 11 from Eq. 10 results in,

$$
\frac{S_{S F G}(R H)}{\sqrt{S_{N R}}}-\frac{S_{S F G}(30.4 \%)}{\sqrt{S_{N R}}} \propto 2 A_{R}(w) \cos (\Delta \varphi(w)) \sqrt{I_{1} I_{2}}
$$

$\Delta \varphi(\mathrm{w})$ is the phase difference between the OD feature and the $\mathrm{S}_{\mathrm{NR}}$. The whole data extraction process is summarized in SI Fig.8

Typically, the phase of molecular spectra is frequency (w) dependent, if the spectra have Lorentzian lineshape; and it is frequency independent when the spectra are inhomogeneously broadened, or have a Gaussian lineshape. As shown in SI Figure 9, the OD features have Gaussian lineshape. Thus, $\Delta \varphi(\mathrm{w})$ should be a constant. From the spectra, $\Delta \varphi(\mathrm{w})$ is around $\pi$, as the spectral feature is a dip. Since $\cos (\Delta \varphi(w))$ and $\sqrt{I_{1} I_{2}}$ are both constants we will only fit $A_{R-}$ (w), the absolute intensity, following a Voigt profile, which is a convolution of Lorentizian (homogeneous broadening) and Gaussian (inhomogeneous broadening) lineshapes.

$$
A_{R}(w)=\sum_{v} \int \frac{A_{v}}{\left(w_{I R}-w_{L}\right) i \Gamma_{L, v}} x e^{-\left(\frac{w_{L}-w_{v}{ }^{2}}{\Gamma_{v}}\right)} d w_{L}
$$

Where $A_{v}, w_{I R}, w_{L}, \Gamma_{L, v}$ and $\Gamma_{\mathrm{v}}$ are the oscillator strength, IR spectral coverage, Lorentzian peak center, dampening constant and Gaussian linewidth respectively. The fitting was carried in MatLab and results are summarized in SI Figure 11. 
Step 1
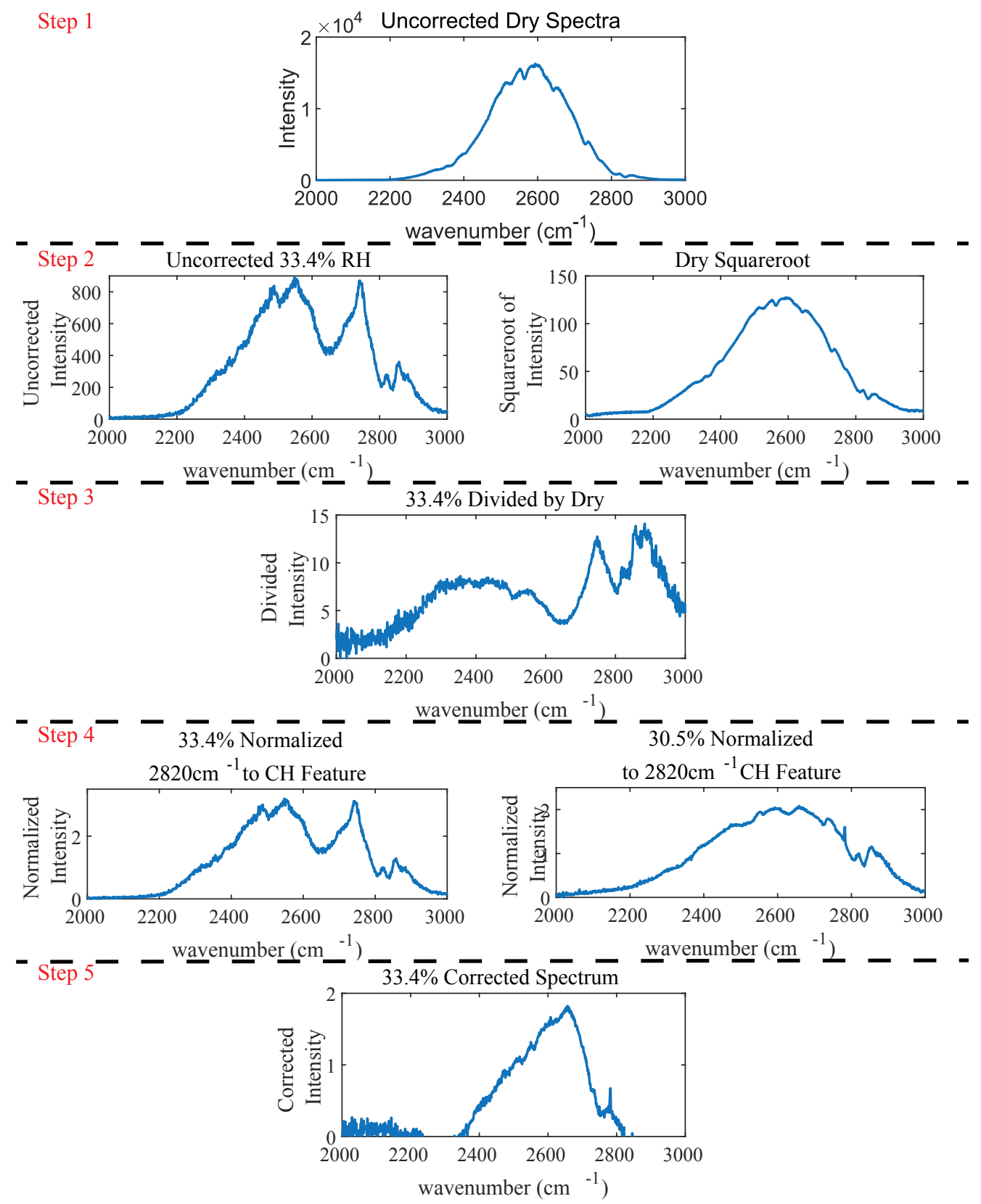

SI Figure 11 Data analysis procedure to extract OD VSFG molecular response. In step 1 the square root of the dry air spectrum is taken to account for the nonresonant response of ZIF-90. In step 2, uncorrected

VSFG spectra are divided by the product of step 1 which accounts for a pseudo-heterodyne signal comprised of molecular responses (CH features and OD features). In step 3, the product of step 2 is normalized to the resonant $\mathrm{CH}$ feature at approximately $2820 \mathrm{~cm}^{-1}$. In step 4 , the spectral difference between $30.4 \%$ and all higher RH spectra to extract the OD molecular response.

\section{S5. VSFG data analysis results}



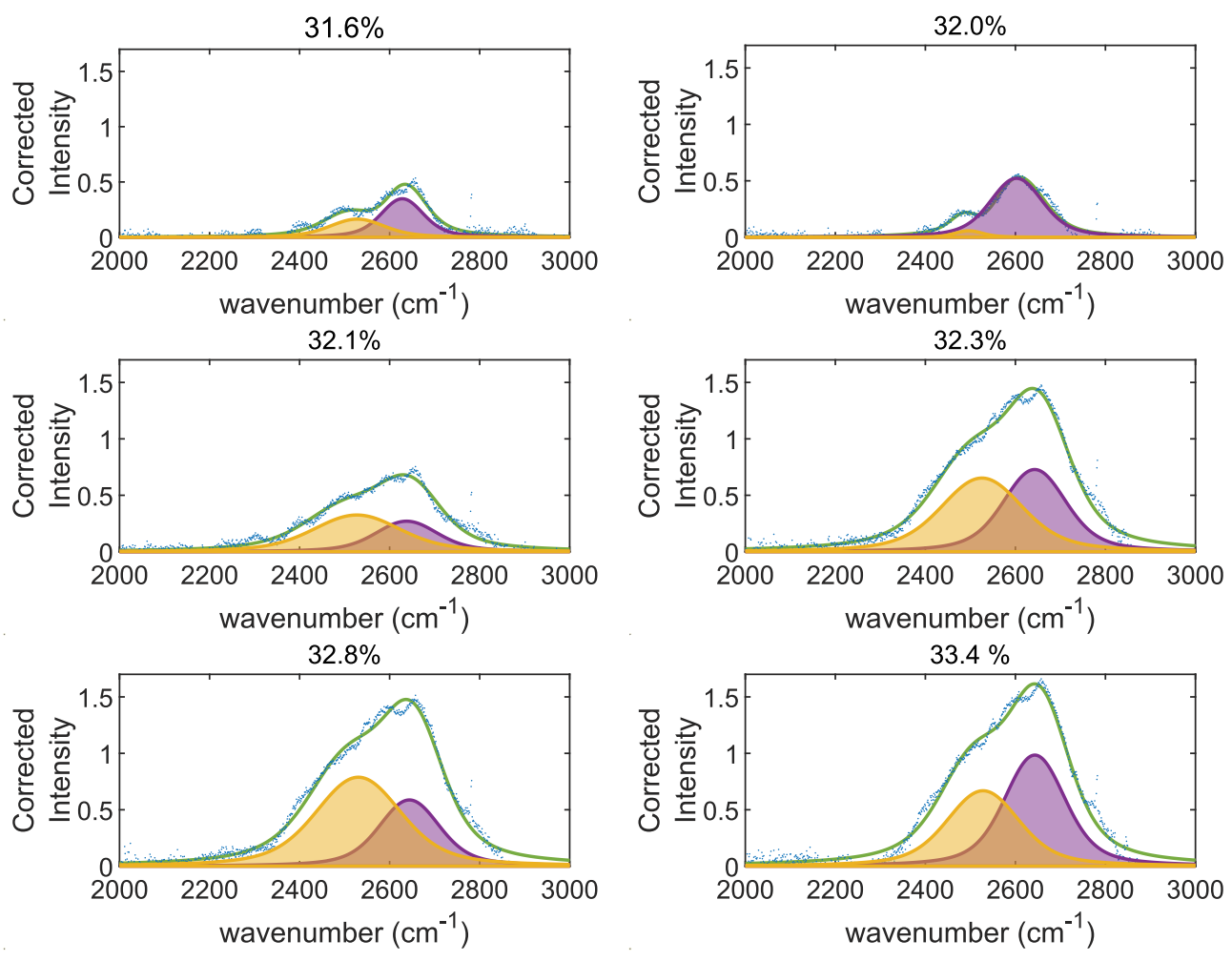

SI Figure 12 Voigt fitting of $\mathrm{D}_{2} \mathrm{O}$ spectral dip feature observed from $31.6 \%$ to $33.4 \% \mathrm{RH}$. Spectra here were first analyzed using the method described in SI S4.

SI Table 2 Relative residual for VSFG Voigt fitting results.

\begin{tabular}{|c|c|c|c|c|c|c|}
\hline$\% \mathrm{RH}$ & $31.60 \%$ & $32.00 \%$ & $32.10 \%$ & $32.30 \%$ & $32.80 \%$ & $33.40 \%$ \\
\hline Relative Residual & $17.9 \%$ & $12.8 \%$ & $11.7 \%$ & $8.4 \%$ & $8.5 \%$ & $8.9 \%$ \\
\hline
\end{tabular}

We did not include the $95 \%$ confidence interval because the Voigt profile used in the VSFG fitting, which is a convolution of a Gaussian and Lorentzian profile, was coded in-house in MatLab. Traditional function solvers in MatLab offer this however, since the Voigt fitting code was manually coded, we were unable to include a $95 \%$ confidence. Instead, we include the relative residual result of the fitting.

\section{S6. Simulation methods and additional results}

The force fields used to model water confined in ZIF-90 are the same as previously described in Ref. 3 for a $2 \times 2 \times 2$ unit cell of ZIF-90.

Simulations of small numbers of water molecules were also performed in a 1x1x1 unit cell so that all the water molecules remained in one pore. As the number of water molecules increases from 2 to 10, the distance of maximum intensity decreases in the $\mathrm{OW}-\mathrm{OW}$ radial distribution function (RDF, Fig. SI 13a). For two and three water molecules, the maximum in the $\mathrm{OW}-\mathrm{OW}$ $\mathrm{RDF}$ is above $2.87 \AA$, but the maximum in the OW-OW RDF is closer to $2.80 \AA$ for simulations with more than 4 water molecules. Additionally, a peak for the second solvation shell of water begins to appear when 6 water molecules are in the pore. As more water is adsorbed in a single pore, the distance of 
maximum intensity in the OW-o RDF decreases (Fig. SI 13b). This information relates that for 2 or 3 water molecules inside the pore, hydrogen bonds are longer and weaker between water molecules themselves and between water and the MOF. However, when more than 4 water molecules, especially when there are more than 6 water molecules in a single pore, hydrogen bonds between water molecules and between water and the MOF are shorter and stronger, as is evidenced by the RDFs (Fig. SI 13).

Therefore, water clusters with short and strong hydrogen bonds begin to appear when there are more than 4 or 6 water molecules in a single pore. Anything larger than 6 water molecules in a single pore can be described as a larger water cluster until the whole pore is filled.
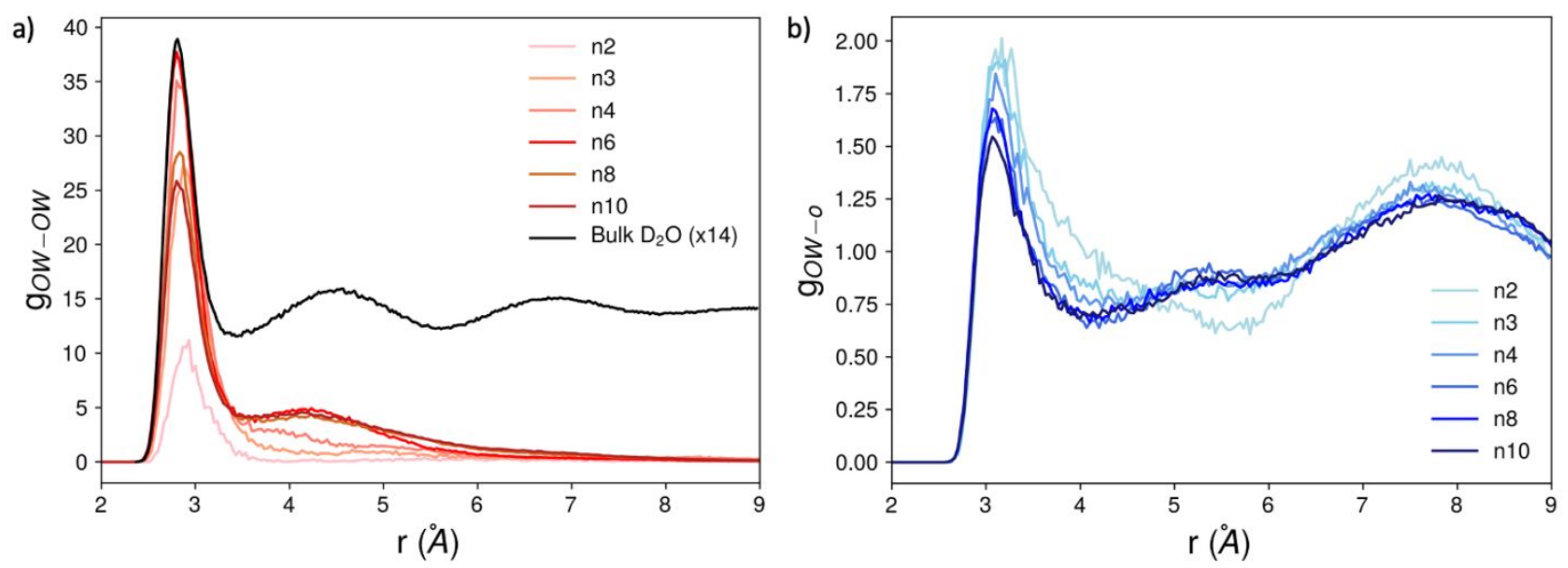

SI Figure 13 a) OW - OW and b) OW - o radial distribution functions (RDFs) for simulations of $\mathrm{nX}$ water molecules (X $=$ total number of water molecules) in a $1 \mathrm{x} 1 \mathrm{x} 1$ simulation cell.

The density of states (DOS) spectra were calculated using

$$
I_{D O S}=\int_{-\infty}^{\infty} e^{i \omega t}\langle v(0) v(t)\rangle
$$

in the time-dependent formalism. In eq. $1,\langle v(0) v(t)\rangle$ is the ensemble-averaged classical velocity-velocity autocorrelation function calculated by averaging $v(0) v(t)$ over all the MD trajectories at each RH. 

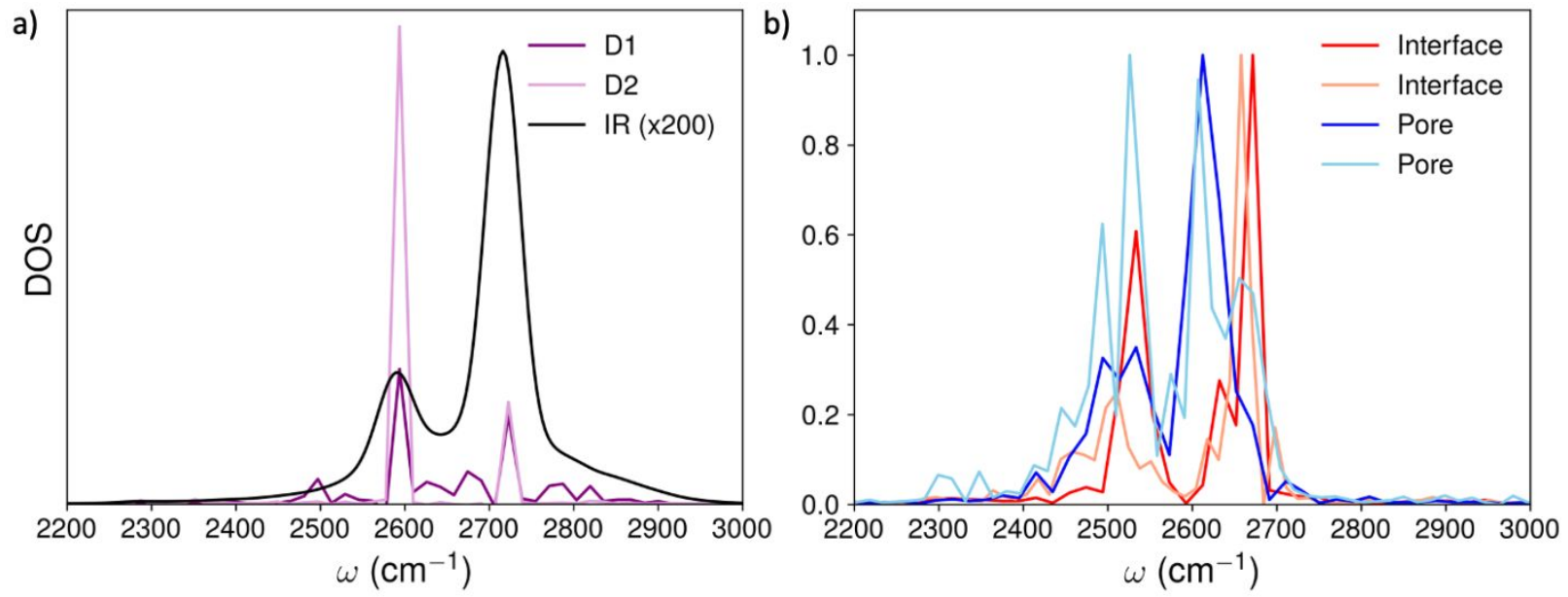

SI Figure 14 a) DOS of a single water molecule at the MOF interface. The two purple lines correspond to the DOS lineshape of each individual deuterium atom in $\mathrm{D}_{2} \mathrm{O}$. The IR lineshape of a single $\mathrm{D}_{2} \mathrm{O}$ is displayed for reference. All lineshapes are red-shifted by $137 \mathrm{~cm}^{-1}$. b) DOS of individual water molecules at the interface vs. in the middle of the pore for a subset of the $40 \% \mathrm{RH}$ simulation. Water at the interface is red (the two red lines are two separate water molecules at the interface), and water in the middle of the pore is blue (the two blue lines are two separate water molecules in the center of the pore). Overall, the lineshapes are similar to the DRIFTS and SFG lineshapes. Both interface water and pore water have two predominant peaks, which resemble the symmetric and asymmetric stretches. Peak positions are slightly red-shifted for water in the middle of the pore and are red-shifted compared to the single water molecule in panel a. All lineshapes are red-shifted by $137 \mathrm{~cm}^{-1}$.

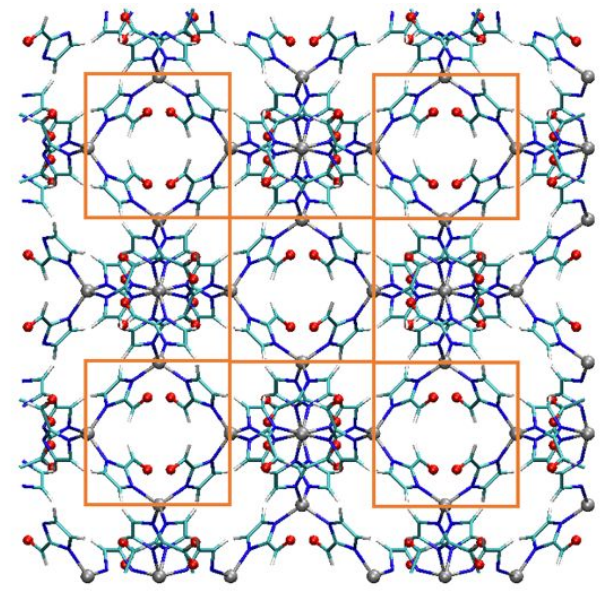

SI Figure 15 Schematic of the ZIF-90 framework used in the simulation; each square indicates pore space where water can be adsorbed. 

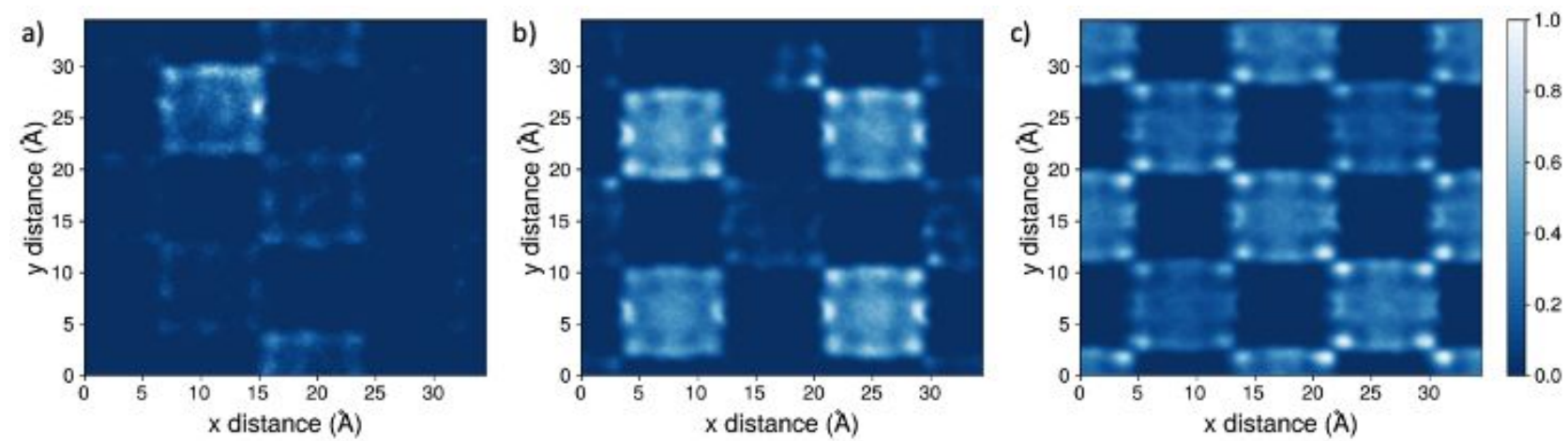

SI Figure 16 Two-dimensional (2D) density plots of water throughout the NVT simulations at (a) $25 \%$ $\mathrm{RH}$, (b) $35 \% \mathrm{RH}$, and (c) 50\% RH. Lighter colors represent regions of higher water density.
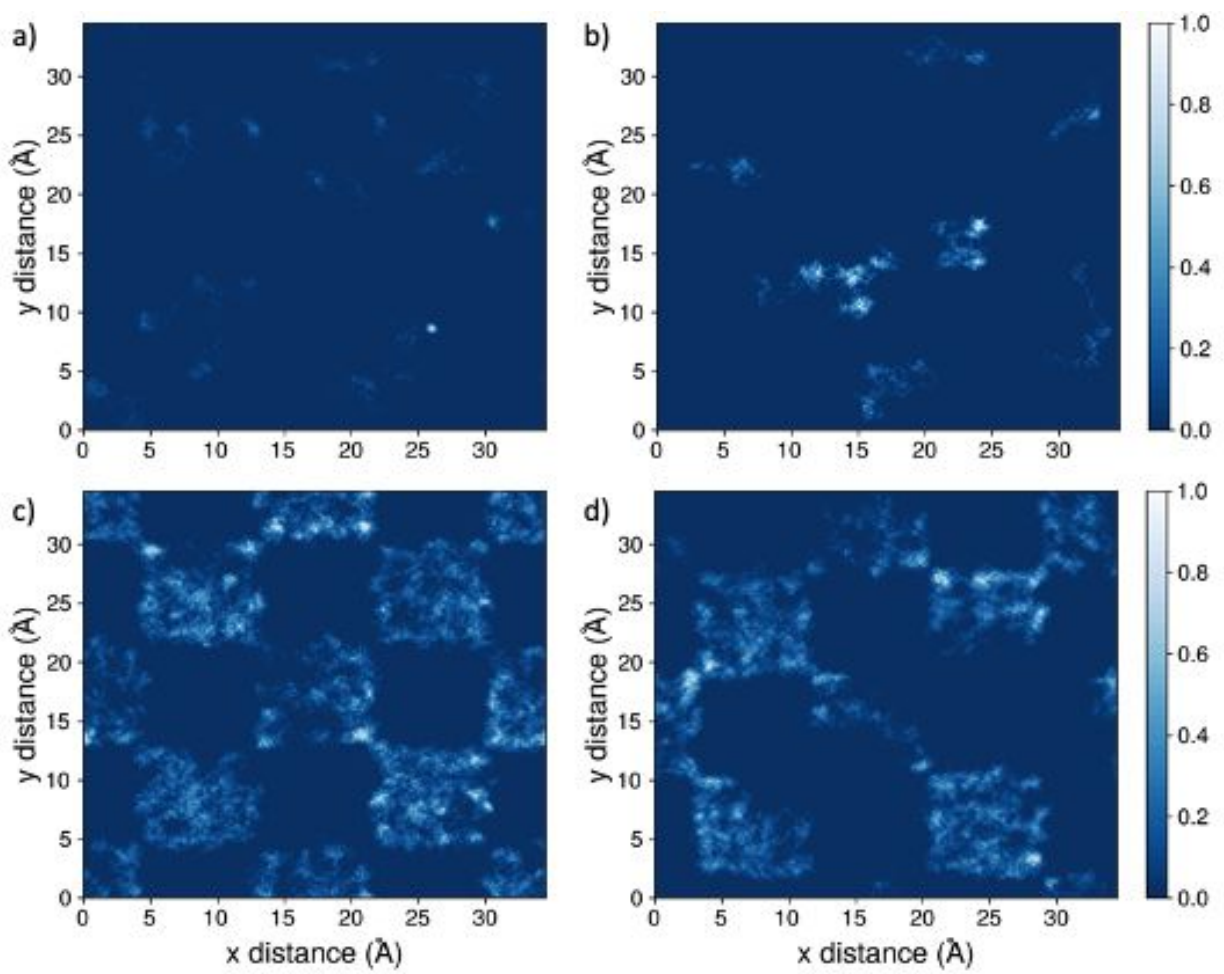

SI Figure $172 \mathrm{D}$ density plots of water in the NVT equilibration simulations. (a) $30 \% \mathrm{RH}$ for $0-10 \mathrm{ps}$ of the NVT simulation. (b) $30 \%$ RH for 990-1000ps of the NVT simulation. (c) $35 \%$ RH for $0-10$ ps of the NVT simulation. (d) 35\% RH for 990-1000ps of the NVT simulation. Panels (a) and (c) show the starting homogeneous distribution of water in the pores, whereas panels (b) and (d) show how water coalesces into fewer pores to interact with itself at the end of the equilibration. Lighter colors represent regions of higher water density. 
The enthalpy of adsorption at each RH was calculated using

$$
\Delta H=\frac{E_{M O F+\text { wat }}-E_{\text {MOF }}-\left(N_{\text {wat }} \times E_{\text {wat }}\right)+\left(N_{\text {wat }} \times R \times T\right)}{N_{\text {wat }}}
$$

where $E_{M O F}+$ wat is the energy of the MOF + water system, $E_{M O F}$ is the energy of the bare MOF, $E_{\text {wat }}$ is the energy of a single MB-pol water molecule, $N_{\text {wat }}$ is the number of water molecules at each RH, R is the gas constant, and $\mathrm{T}$ is the system temperature.

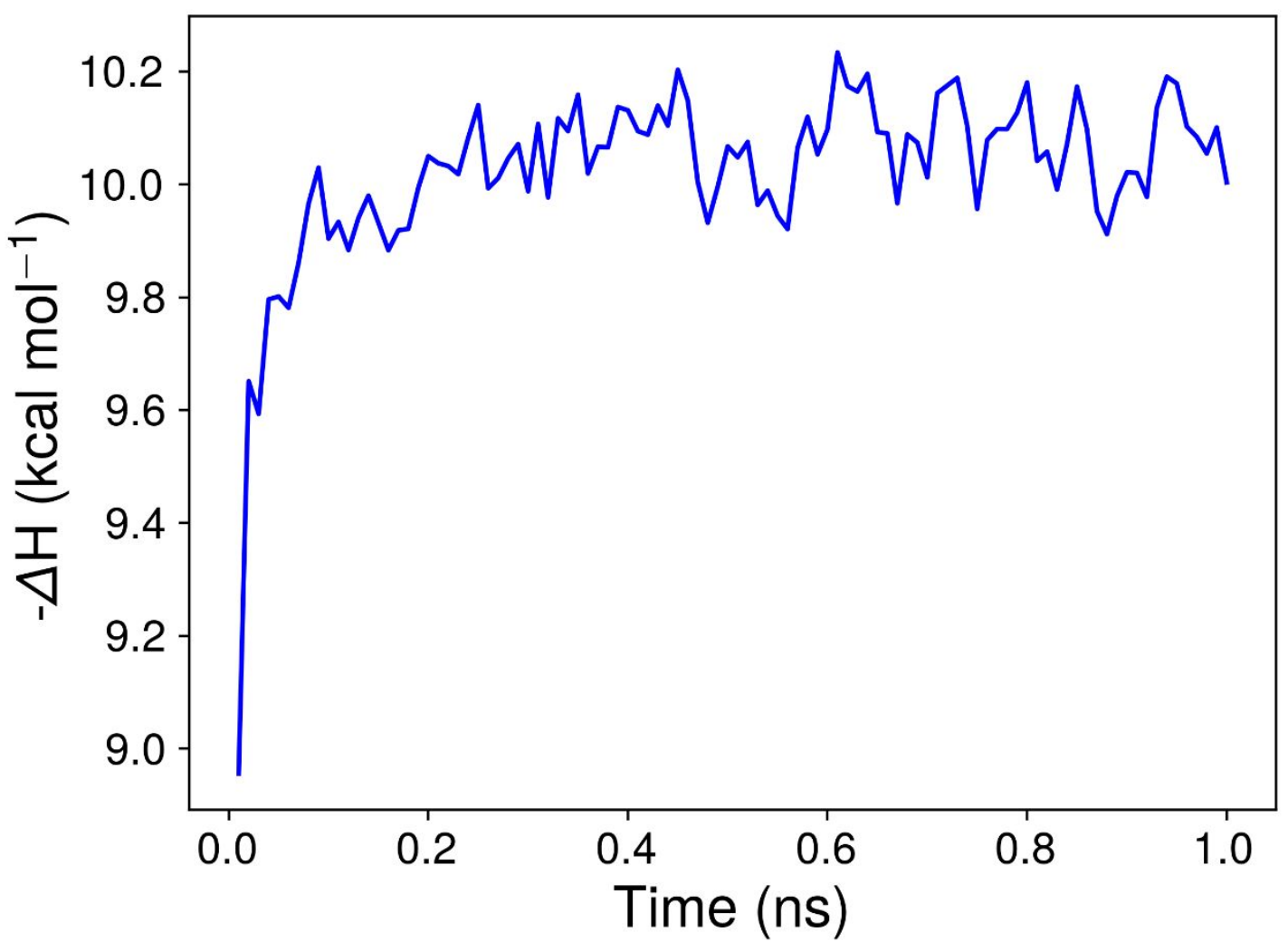

SI Figure 18 Enthalpy of adsorption at 35\% RH. Each point represents an average over $10 \mathrm{ps}$ of the simulation.

The entropy of water confined in ZIF-90 was calculated using the Two-Phase Thermodynamics (2PT) method. ${ }^{3-5}$ Water confined in ZIF-90 has slower orientational dynamics compared to bulk water (SI Tables 4 and 6) and thus has a higher entropy than bulk water. The excess entropy $\left(\mathrm{S}^{\mathrm{exc}}\right)$ of $\mathrm{D}_{2} \mathrm{O}$ confined in ZIF-90 is calculated in Table 3 as: 


$$
S^{e x c}=S_{\text {wat in } M O F}-S_{\text {bulk wat }}
$$

SI Table 3 Excess entropy of $\mathrm{D}_{2} \mathrm{O}$ confined in ZIF-90 at various RH. The entropy of simulated bulk $\mathrm{D}_{2} \mathrm{O}$ is $65.21 \mathrm{~J} / \mathrm{mol} / \mathrm{K} /$ molecule, and the entropy of experimental bulk $\mathrm{D}_{2} \mathrm{O}$ is $75.64 \mathrm{~J} / \mathrm{mol} / \mathrm{K} / \mathrm{molecule}$ from Ref. $6^{6}$.

\begin{tabular}{|c|c|}
\hline $\mathrm{RH}$ & $\mathrm{S}^{\mathrm{exc}}(\mathrm{J} / \mathrm{mol} / \mathrm{K} / \mathrm{molecule})$ \\
\hline $25 \%$ & 40.50 \\
\hline $30 \%$ & 31.16 \\
\hline $35 \%$ & 11.10 \\
\hline $40 \%$ & 5.35 \\
\hline $45 \%$ & 7.25 \\
\hline $50 \%$ & 5.26 \\
\hline $60 \%$ & 5.48 \\
\hline $70 \%$ & 4.94 \\
\hline
\end{tabular}

The orientational correlation functions were calculated using

$$
C_{2}(t)=\left\langle P_{2}[e(0) \cdot e(t)]\right\rangle
$$

where $P_{2}[e(0) e(t)]$ is the second Legendre polynomial of the angle formed by the unit vector $e(t)$ that lies along one of the OD bonds of a water molecule. The angle brackets suggest an ensemble average of OD bonds over time. 

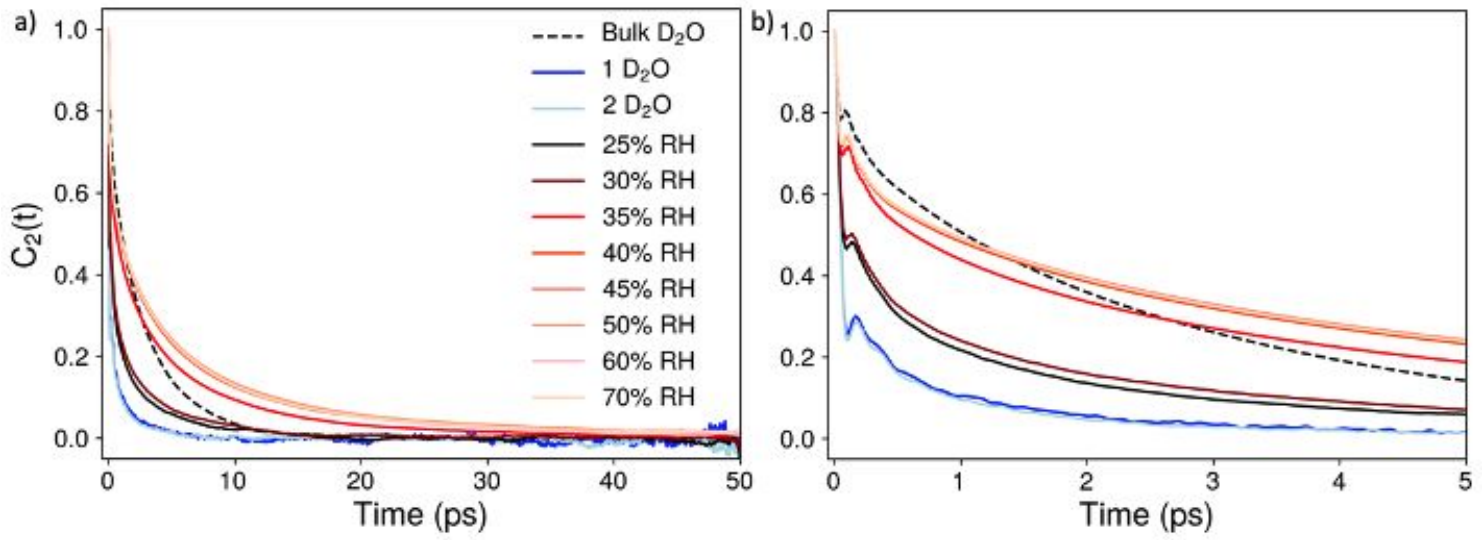

SI Figure 19 (a) Orientational correlation functions for bulk $\mathrm{D}_{2} \mathrm{O}$ and water confined in ZIF-90 at various RH. (b) Orientational correlation functions at short simulation time. Colors are the same as in panel (a). form

The orientational correlation functions from Fig. S19 were fit to a triexponential function of the

$$
C_{2}(t)=A_{1} e^{-\frac{t}{\tau_{1}}}+A_{2} e^{-\frac{t}{\tau_{2}}}+A_{3} e^{-\frac{t}{\tau_{3}}}
$$

where $A_{1}, A_{2}$, and $A_{3}$ are constants, and $\tau_{1}, \tau_{2}$, and $\tau_{3}$ are the relaxation time constants of different motions, with $\tau_{2}$ corresponding to the hydrogen bond rearrangement time.

SI Table 4 Relaxation time constants for the orientational correlation functions at various RH and bulk $\mathrm{D}_{2} \mathrm{O}$.

\begin{tabular}{|c|c|c|c|}
\hline $\mathrm{RH}$ & $\tau_{1}(\mathrm{ps})$ & $\tau_{2}(\mathrm{ps})$ & $\tau_{3}(\mathrm{ps})$ \\
\hline $25 \%$ & 0.03 & 0.63 & 4.51 \\
\hline $30 \%$ & 0.03 & 0.71 & 5.23 \\
\hline $35 \%$ & 0.22 & 2.97 & 12.45 \\
\hline $40 \%$ & 0.23 & 3.72 & 16.78 \\
\hline $45 \%$ & 0.23 & 3.80 & 17.13 \\
\hline $50 \%$ & 0.25 & 3.97 & 18.96 \\
\hline $60 \%$ & 0.19 & 3.24 & 13.78 \\
\hline $70 \%$ & 0.26 & 4.03 & 18.10 \\
\hline Simulated bulk $\mathrm{D}_{2} \mathrm{O}$ & 0.02 & 0.70 & 3.48 \\
\hline
\end{tabular}


a)

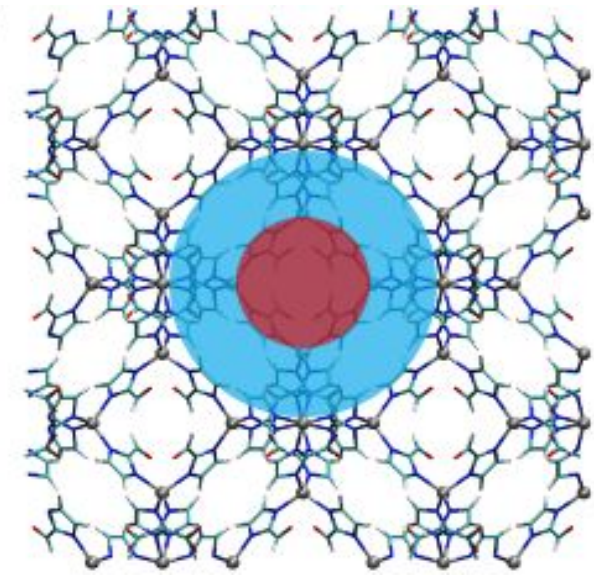

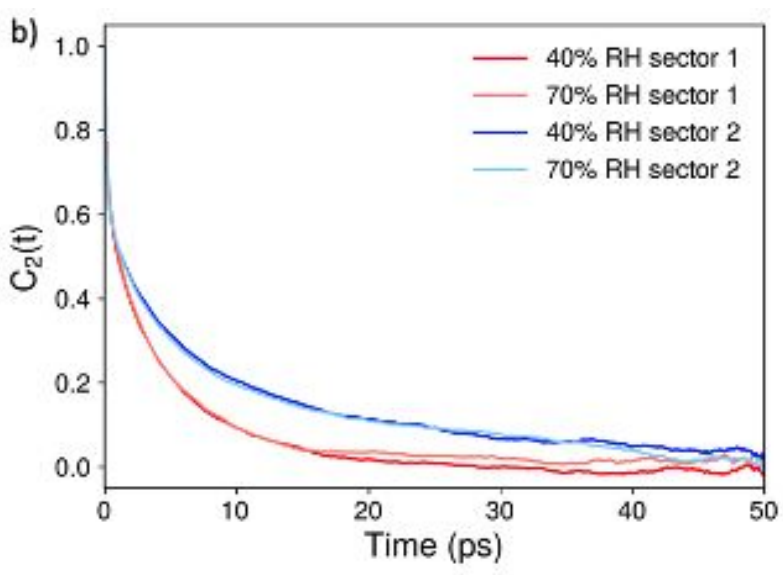

SI Figure 20 (a) ZIF-90 pore split into two sectors. Sector 1 (red) is in the middle of the pore with no interaction with the MOF interface, and sector 2 (blue) is the region near the MOF interface. (b) Orientational correlation functions for water at 40\% RH (darker colors) and 70\% RH (lighter colors) in sector 1 (red colors) and sector 2 (blue colors).

The orientational correlation functions of different sectors were fit to a triexponential function of the form from SI equation 13 where $A_{1}, A_{2}$, and $A_{3}$ are constants, and $\tau_{1}, \tau_{2}$, and $\tau_{3}$ are the relaxation time constants of different motions, with $\tau_{2}$ corresponding to the hydrogen bond rearrangement time.

SI Table 5 Relaxation time constants for the orientational correlation functions of different sectors.

\begin{tabular}{|c|c|c|c|}
\hline RH & $\tau_{1}(\mathrm{ps})$ & $\tau_{2}(\mathrm{ps})$ & $\tau_{3}(\mathrm{ps})$ \\
\hline $40 \%$ sector 1 & 0.03 & 0.75 & 5.77 \\
\hline $70 \%$ sector 1 & 0.25 & 3.67 & 14.32 \\
\hline $40 \%$ sector 2 & 0.16 & 4.30 & 24.29 \\
\hline $70 \%$ sector 2 & 0.14 & 3.26 & 17.67 \\
\hline
\end{tabular}

The diffusion coefficients were calculated using

$$
D=\frac{1}{3} \int_{0}^{\infty}\langle v(0) v(t)\rangle d t
$$

where $\langle v(0) v(t)\rangle$ is the ensemble-averaged classical velocity-velocity autocorrelation function. The limit of the autocorrelation function at long times is taken to determine the total diffusion coefficient.

SI Table 6 Total diffusion coefficient, total diffusion coefficient for sector 1, and total diffusion coefficient for sector 2 at various RH. No diffusion coefficient is reported for $35 \%$ RH sector 1 as there were not enough water molecules in this region to calculate the diffusion coefficient.

\begin{tabular}{|c|c|c|c|}
\hline RH & $\mathrm{D}_{\text {tot }}\left(\AA^{2} \cdot \mathrm{ps}^{-1}\right)$ & $\begin{array}{c}\text { Sector } 1 \\
\mathrm{D}_{\text {tot }}\left(\AA^{2} \cdot \mathrm{ps}^{-1}\right)\end{array}$ & $\begin{array}{c}\text { Sector } 2 \\
\mathrm{D}_{\text {tot }}\left(\AA^{2} \cdot \mathrm{ps}^{-1}\right)\end{array}$ \\
\hline $35 \%$ & 0.15 & $\mathrm{~N} / \mathrm{A}$ & 0.05 \\
\hline $40 \%$ & 0.11 & 0.11 & 0.06 \\
\hline $45 \%$ & 0.10 & 0.07 & 0.06 \\
\hline $50 \%$ & 0.09 & 0.09 & 0.06 \\
\hline
\end{tabular}




\begin{tabular}{|c|c|c|c|}
\hline $60 \%$ & 0.11 & 0.09 & 0.03 \\
\hline $70 \%$ & 0.10 & 0.11 & 0.04 \\
\hline Simulated bulk $\mathrm{D}_{2} \mathrm{O}$ & 0.17 & & \\
\hline
\end{tabular}

The H-bond correlation function was calculated using

$$
c(t)=\frac{\langle h(0) h(t)\rangle}{\langle h\rangle}
$$

where $\langle h(0) h(t)\rangle$ is the ensemble-averaged hydrogen-bond correlation function. The function $h(t)$ has a value of 0 if water does not form a H-bond to the aldehyde group of the linker at time $t$, and $h(t)$ has a value of 1 if water forms a $\mathrm{H}$-bond to the aldehyde group of the linker at time $t .\langle h\rangle$ is the average of the H-bond correlation.

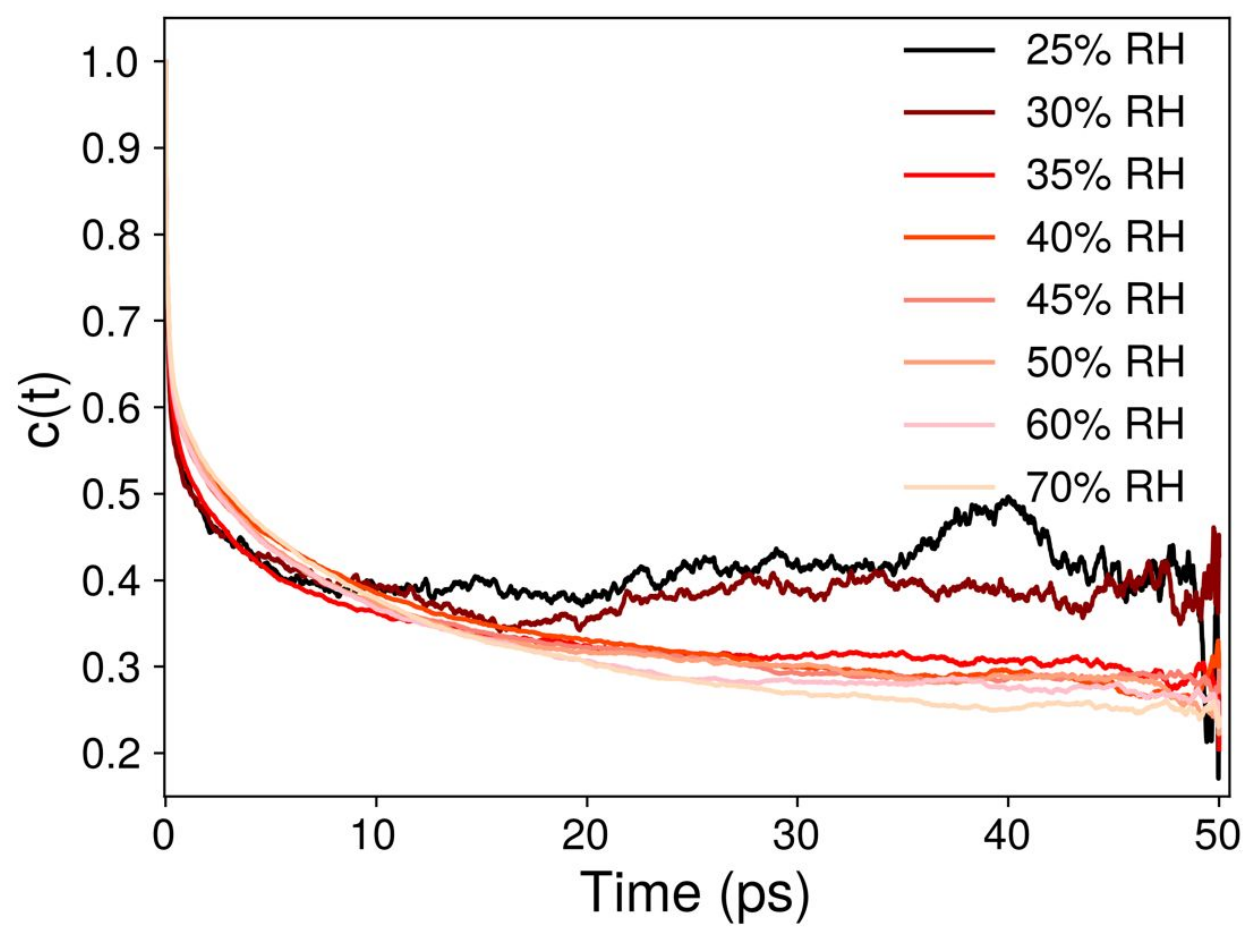

SI Figure 21 H-bond correlation function for water at various $R H$.

The H-bond correlation functions were fit to a triexponential function of the form from SI equation 13 where $A_{1}, A_{2}$, and $A_{3}$ are constants, and $\tau_{1}, \tau_{2}$, and $\tau_{3}$ are the relaxation time constants of different motions with $\tau_{2}$ representing the hydrogen bond lifetime. 
SI Table 7 Relaxation time constants for the H-bond correlation function.

\begin{tabular}{|c|c|c|c|}
\hline $\mathrm{RH}$ & $\tau_{1}(\mathrm{ps})$ & $\tau_{2}(\mathrm{ps})$ & $\tau_{3}(\mathrm{ps})$ \\
\hline $25 \%$ & 0.078 & 1.55 & $>1000$ \\
\hline $30 \%$ & 0.112 & 2.97 & $>1000$ \\
\hline $35 \%$ & 0.155 & 4.31 & 289.9 \\
\hline $40 \%$ & 0.162 & 5.96 & 179.1 \\
\hline $45 \%$ & 0.167 & 6.39 & 308.1 \\
\hline $50 \%$ & 0.136 & 5.28 & 202.4 \\
\hline $60 \%$ & 0.185 & 7.40 & 443.9 \\
\hline $70 \%$ & 0.190 & 8.58 & 303.4 \\
\hline
\end{tabular}

\section{Supporting References}

(1) Yang, T.; Chung, T. S. Room-Temperature Synthesis of ZIF-90 Nanocrystals and the Derived NanoComposite Membranes for Hydrogen Separation. J. Mater. Chem. A 2013, 1 (19), 6081-6090. https://doi.org/10.1039/c3ta10928c.

(2) Eum, K.; Jayachandrababu, K. C.; Rashidi, F.; Zhang, K.; Leisen, J.; Graham, S.; Lively, R. P.; Chance, R. R.; Sholl, D. S.; Jones, C. W.; Nair, S. Highly Tunable Molecular Sieving and Adsorption Properties of Mixed-Linker Zeolitic Imidazolate Frameworks. J. Am. Chem. Soc. 2015, 137 (12), 4191-4197. https://doi.org/10.1021/jacs.5b00803.

(3) Pascal, T. A.; Lin, S. T.; Goddard, W. A. Thermodynamics of Liquids: Standard Molar Entropies and Heat Capacities of Common Solvents from 2PT Molecular Dynamics. Phys. Chem. Chem. Phys. 2011, 13 (1), 169-181. https://doi.org/10.1039/c0cp01549k.

(4) Lin, S. T.; Maiti, P. K.; Goddard, W. A. Two-Phase Thermodynamic Model for Efficient and Accurate Absolute Entropy of Water from Molecular Dynamics Simulations. J. Phys. Chem. B 2010, 114 (24), 8191-8198. https://doi.org/10.1021/jp103120q.

(5) Phys, J. C.; Lin, S.; Blanco, M.; Goddard, W. A. The Two-Phase Model for Calculating Thermodynamic Properties of Liquids from Molecular Dynamics : Validation for the Phase Diagram of Lennard-Jones Fluids. 2017, 11792 (2003). https://doi.org/10.1063/1.1624057.

(6) Némethy, G.; Scheeaga, H. A. Structure of Water and Hydrophobic Bonding in Proteins. I. A Model for the Thermodynamic Properties of Liquid Water. J. Chem. Phys. 1962, 36 (12), 33823400. https://doi.org/10.1063/1.1732472. 